\title{
Monocyte subpopulations study in patients with plaque psoriasis
}

\author{
Mariana Carvalho Costa ${ }^{\mathrm{a}, *}$, Bruno de Oliveira Rocha ${ }^{\mathrm{b}}$, Camilla Sampaio Paixão ${ }^{\mathrm{b}}$, \\ Maria de Fátima Santos Paim de Oliveira ${ }^{\mathrm{c}}$, Lícia Maria Henrique da Mota ${ }^{\mathrm{a}}$, Lucas Pedreira de Carvalho ${ }^{\mathrm{b}, \mathrm{d}}$ \\ ${ }^{a}$ Faculty of Medicine, University of Brasília, Brazil \\ ${ }^{\mathrm{b}}$ Immunology Service, Prof. Edgard Santos University Hospital, Federal University of Bahia, Brazil \\ ${ }^{\mathrm{c}}$ Department of Dermatology, Federal University of Bahia, Brazil \\ ${ }^{\mathrm{d}}$ Gonçalo Moniz Institute, Fiocruz, Salvador, BA, Brazil
}

\section{A R T I C L E I N F O}

\section{Article history:}

Received 9 October 2016

Accepted 25 May 2017

\begin{abstract}
A B S T R A C T
Psoriasis is a chronic and systemic, immune-mediated, inflammatory disease, mainly manifested by skin and / or joints lesions, presenting with a wide degree of clinical severity, but generally with great impact on patients' quality of life. Despite advances in the understanding of its pathogenesis, especially regarding the participation of T-lymphocytes and the key role of TNF, the triggering factor of the disease at the molecular level remains unknown, as well as the function of other cell populations. By presenting antigens to T-lymphocytes, monocytes assume an important role in both innate and adaptive immune response. In the last two decades, by using flow cytometry with antibodies against CD14 (receptor for lipopolysaccharide) and CD16 (low affinity receptor for $\operatorname{IgG}$ ), human blood monocytes were classified into three subpopulations: classical (CD14++CD16-), intermediate (CD14++CD16+), and non-classical (CD14 $+\mathrm{CD} 16++$ ). Under normal conditions the population of classical monocytes corresponds to about $85 \%$, while intermediate to $5.4 \%$, and nonclassical to $9.2 \%$. However, intermediate and nonclassical subsets are increased in various inflammatory situations, such as moderate to severe asthma, colorectal cancer, and rheumatoid arthritis. Despite psoriasis being considered a disease of inflammatory nature, scarce studies evaluating the frequency of subpopulations of monocytes in psoriatic patients are found on current medical literature, and they are restricted to peripheral blood analysis. This study aims to identify the frequency of monocyte subpopulations in blood levels as well as lesional skin of plaque psoriasis patients, and to evaluate their association to cytokines, and clinical disease severity.
\end{abstract}

(c) 2017 Elsevier Ltd. All rights reserved.

\section{Introduction background}

Psoriasis is a chronic, systemic, immune-mediated, inflammatory disease, mainly manifested by skin and/or joints lesions, that affects about $2-3 \%$ of the adults worldwide [1]. In the skin, the most common presentation, called plaque psoriasis, is characterized by erythematous, scaly, infiltrated, and well-delimited papules/plaques, varying on size, and symmetrically distributed [2]. Besides skin features, about $20-30 \%$ of psoriasis patients develop psoriatic arthritis, which can result in irreversible deformities [1]. Both cutaneous and arthropathic involvements interfere dramatically on patients' quality of life [3].

Despite advances in the understanding of its pathogenesis, molecular triggering factors of the disease remain unknown, as well as the function of some cell populations. It is believed that after stimulation of antigen-presenting cells (APC) and activation

* Corresponding author at: SHLS 716 - Centro Clínico Sul - Torre II - sala 18, 70390-700 Brasília, DF, Brazil

E-mail address: maricosta133@gmail.com (M.C. Costa). of T-cells, release of cytokines occurs, specially interleukin 1 (IL1) and tumor necrosis factor (TNF) [4]. Concerning adaptive immune response, the prominent response is Th1 (T-helper 1) type, with increased expression of interferon- $\gamma$ (INF- $\gamma$ ), TNF, IL-2, and IL-12 in skin lesions of psoriasis. These cytokines induce epidermal and blood vessels hyperproliferation and other proinflammatory effects that are more evident in patients with more severe disease $[5,6]$. Several cascading reactions occur with the release of these inflammatory mediators. For example, TNF induces expression of IL- 6 and C-reactive protein. IL-6 mediates T-cell activation and acute phase response, and stimulates the proliferation of keratinocytes. The release of both IFN- $\gamma$ and TNF also activates keratinocytes, which begins secreting IL-8, which has potent chemotactic activity for neutrophils [7]. Within the epidermis, neutrophils release mediators that act on endothelial cells, which in turn express adhesion molecules. This allows an increasing influx of leukocytes that perpetuates the inflammatory process. It is noteworthy that TNF plays a key role in the pathogenesis of psoriasis, being one of the first cytokines secreted by a large number of 
cells: macrophages, T-cells, natural killer (NK) cells, and keratinocytes. It is no coincidence the therapeutic effectiveness of inhibitory drugs of this cytokine [8].

More recently, studies that have pointed to the importance of Th17 and Th22 immune responses on psoriasis. The IL-23 expression, cytokine secreted by keratinocytes, Langerhans cells, macrophages, and dendritic cells are increased in psoriatic lesions and is involved in the secretion of IL-17 by Th17 cells [9]. Th22 cells are a new population of lymphocytes described and express IL22 and TNF [4]. Despite that, the T-cells are not unique in the pathogenesis of psoriasis. Other cell populations influence at different levels regarding its pathogenesis and intensity of clinical features [10]. Monocytes, mononuclear leukocytes are derived from a common precursor myeloid bone marrow, are differentiated into macrophages and dendritic cells in the tissues, and are known by their phagocytic and antigen-presenting capacity. By presenting antigens to T-lymphocytes, monocytes assume an important role in both innate and adaptive immune response [11]. In psoriasis lesions, the infiltration of monocytes and T-cells is well-described, and it is assumed that Th1 and Th17 cells interact with monocytes, inducing their differentiation into specialized dendritic cells [12]. Additionally monocytes are the main IL-1, IL6 and TNF producing-cells, cytokines of major importance in psoriasis $[10,12]$.

In the last two decades, by using flow cytometry with antibodies against CD14 (receptor for lipopolysaccharide) and CD16 (low affinity receptor for IgG), human blood monocytes were classified primarily into two subpopulations, which have different functional and phenotypic characteristics, based on CD16 expression: CD14 + +CD16 - subset, and CD14+CD16+ subset $[13,14]$. Several subsequent studies, however, have identified heterogeneity on the CD16+ subgroup, with an intermediate subpopulation associated to relatively higher expression of CD14 and lower expression of CD16, and another nonclassical subpopulation expressing relatively lower levels of CD14 associated with higher expression of CD16. Thus, a new nomenclature was proposed, by grouping these cells into three subpopulations: 1 ) CD14++ CD16- (high expression of CD14 without the expression of CD16, termed classical monocytes); 2) CD14++ CD16+ (high expression of CD14 with low expression of CD16, termed intermediate monocytes); and 3) CD14+ CD16++ (low expression of CD14 and high CD16 expression, non-classical monocytes) $[13,15]$. In terms of percentage, it is believed that, under normal conditions, the population of classical monocytes corresponds to about $85 \%$, while the intermediate to $5.4 \%$, and the nonclassical to $9.2 \%$ [16]. Currently, it remains unclear whether intermediate monocytes represent only one type of cell in transition or actually constitute a distinct cell population [17]. From the clinical point of view, intermediate monocytes are increased in various inflammatory conditions, such as moderate to severe asthma [18], colorectal cancer [19], and rheumatoid arthritis [20]. In the latter, high frequency of intermediate monocytes was also associated with decreased response to methotrexate therapy [20]. Studies also relate intermediate monocytes as predictors of poor outcome in patients with cardiovascular disease $[21,22]$.

So far, though considered a disease of inflammatory nature, there are very few studies evaluating the frequency of monocytes subpopulations in patients suffering from psoriasis, and are restricted to peripheral blood analysis $[23,24]$.

\section{The hypothesis/theory}

Being psoriasis an immune-mediated disease, variations in the frequency of monocytes subpopulations, which indicates inflammatory activity, are expected to be found in peripheral blood and lesional skin of psoriasis patients. More specifically, the frequency of classical monocytes is supposed to be decreased, while the frequency of intermediate and nonclassical ones (called inflammatory monocytes) would be increased. Still, it is believed to be observed a positive relationship between the rise in inflammatory monocytes subsets and the expression of pro-inflammatory cytokines, as well the clinical severity of the disease measured by Psoriasis Area and Severity Index (PASI).

\section{Evaluation of the hypothesis/idea}

To investigate this hypothesis, peripheral blood samples and punch skin biopsy of psoriasis lesions will be collected, followed by processing and analysis by flow cytometry. Additionally, through the evaluation of cell surface molecules and cytokines, and the relation with the clinical severity measured by PASI, the hypothesis might be refuted or not. All patient data will be compared to controls findings.

\section{Importance of the hypothesis and discussion}

There are few published data on monocyte subpopulations analysis in patients with psoriasis till the present date. Whereas TNF plays fundamental role in psoriasis and monocytes are one of the major cellular sources of TNF, the proposal to evaluate subpopulations of monocytes in psoriasis in peripheral blood and at cutaneous level will certainly contribute to a better understanding of the disease. It is also possible that the intermediate monocyte frequency as well as pro-inflammatory cytokines production by these cells to be directly correlated with the severity of the disease.

\section{Sources of support}

None.

\section{Conflict of interest}

None.

\section{Acknowledgements}

The authors would like to thank the Department of Dermatology of Prof. Edgard Santos University Hospital/UFBA, specially the following colleagues: Gleison Duarte, MD, PhD, Ivonise Follador, $\mathrm{MD}$, PhD, Vitoria Regina Pedreira Rego, MD, MSc, and Paulo Roberto Machado, MD, PhD.

The authors would also like to greatly thank Prof. Edgar Marcelino de Carvalho Filho, MD, PhD, a remarkable physician, professor and researcher, for the support since the beginning of this project.

\section{References}

[1] Parisi R, Symmons DPM, Griffiths CEM, Ashcroft DM. Global epidemiology of psoriasis: a systematic review of incidence and prevalence. J Invest Dermatol 2012;133(2):377-85.

[2] Boehncke W-H, Boehncke S. More than skin-deep: the many dimensions of the psoriatic disease. Swiss Med Wkly 2014;144:w13968.

[3] Hawro T, Zalewska A, Hawro M, Kaszuba A, Krõlikowska M, Maurer M. Impact of psoriasis severity on family income and quality of life. J Eur Acad Dermatol Venereol 2015;29(3):438-43.

[4] Mitra A, Fallen RS, Lima HC. Cytokine-based therapy in psoriasis. Clin Rev Allergy Immunol 2013;44:173-82.

[5] Arican O, Aral M, Sasmaz S, Ciragil P. Serum levels of TNF-alpha, IFN-gamma, IL-6, IL-8, IL-12, IL-17, and IL-18 in patients with active psoriasis and correlation with disease severity. Mediat Inflamm 2005;2005(5):273-9.

[6] Jacob SE, Nassiri M, Kerdel FA, Vincek V. Simultaneous measurement of multiple Th1 and Th2 serum cytokines in psoriasis and correlation with disease severity. Mediat Inflamm 2003;12(5):309-13.

[7] Tonel G, Conrad C. Interplay between keratinocytes and immune cells-Recent insights into psoriasis pathogenesis. Int J Biochem Cell Biol 2009;41:963-8. 
[8] Lowes MA, Bowcock AM, Krueger JG. Pathogenesis and therapy of psoriasis. Nature 2007;445(7130):866-73.

[9] Piskin G, Sylva-Steenland RMR, Bos JD, Teunissen MBM. In vitro and in situ expression of IL-23 by keratinocytes in healthy skin and psoriasis lesions: enhanced expression in psoriatic skin. J Immunol 2006;176(3):1908-15.

[10] Coimbra S, Figueiredo A, Castro E, Rocha-Pereira P, Santos-Silva A, Santos Silva A. The roles of cells and cytokines in the pathogenesis of psoriasis.

[11] Geissmann F, Manz MG, Jung S, Sieweke MH, Merad M, Ley K. Development of monocytes, macrophages, and dendritic cells. Science 2010;327:656-61.

[12] Ueno H, Klechevsky E, Morita R, Aspord C, Cao T, Matsui T, et al. Dendritic cell subsets in health and disease. Immunol Rev 2007;219:118-42.

[13] Wong KL, Yeap WH, Tai JJY, Ong SM, Dang TM, Wong SC. The three human monocyte subsets: Implications for health and disease. Immunol Res 2012;53 $(1-3): 41-57$.

[14] Wang G, Xia Z. Monocyte subsets and their differentiation tendency after burn injury. Front Med China 2013;7:397-400.

[15] Ziegler-Heitbrock L, Ancuta P, Crowe S, Dalod M, Grau V, Hart DN, et al Nomenclature of monocytes and dendritic cells in blood. Blood 2010;116.

[16] Wong KL, Tai JJ-Y, Wong W-C, Han H, Sem X, Yeap W-H, et al. Gene expression profiling reveals the defining features of the classical, intermediate, and nonclassical human monocyte subsets. Blood 2011;118(5):e16-31.

[17] Ziegler-Heitbrock L, Hofer TPJ, Poli G. Toward a refined definition of monocyte subsets. 2010
[18] Kowal K, Moniuszko M, Dabrowska M, Bodzenta-Lukaszyk A. Allergen challenge differentially affects the number of circulating monocyte subsets. Scand J Immunol 2012;75(5):531-9.

[19] Schauer D, Starlinger P, Reiter C, Jahn N, Zajc P, Buchberger E, et al. Intermediate monocytes but not TIE2-expressing monocytes are a sensitive diagnostic indicator for colorectal cancer. PLoS One 2012;7(9).

[20] Wijngaarden S, van Roon JAG, Bijlsma JWJ, van de Winkel JGJ, Lafeber FPJG. Fcgamma receptor expression levels on monocytes are elevated in rheumatoid arthritis patients with high erythrocyte sedimentation rate who do not use anti-rheumatic drugs. Rheumatology 2003;42(5):681-8.

[21] Rogacev KS, Cremers B, Zawada AM, Seiler S, Binder N, Ege P, et al. CD14+ +CD16+ monocytes independently predict cardiovascular events: a cohort study of 951 patients referred for elective coronary angiography. J Am Coll Cardiol 2012;60(16):1512-20.

[22] Heine GH, Ortiz A, Massy ZA, Lindholm B, Wiecek A, Martinez-Castelao A, et al. Monocyte subpopulations and cardiovascular risk in chronic kidney disease. Nat Rev Nephrol 2012;8(6):362-9.

[23] Kouris A, Pistiki A, Katoulis A, Georgitsi M, Giatrakou S, Papadavid E, et al. Proinflammatory cytokine responses in patients with psoriasis. Eur Cytokine Netw 2014;25(4):63-8.

[24] Fujisawa T, Murase K, Kanoh H, Takemura M, Ohnishi H, Seishima M. Adsorptive depletion of CD14 proinflammatory monocyte phenotype in patients with generalized pustular psoriasis: clinical efficacy and effects on cytokines. 